\title{
Differential sensitivity of GABAergic and glycinergic inputs to orexin-A in preganglionic cardiac vagal neurons of newborn rats ${ }^{1}$
}

\author{
Ji-jiang WANG ${ }^{2}$,Yong-hua CHEN, Ke-yong LI, Feng-yan SUN \\ National Key Laboratory of Medical Neurobiology, Fudan University Shanghai Medical College, Shanghai 200032, China
}

\author{
Key words \\ patch-clamp techniques; nervous system; \\ synaptic transmission; vagus nerve

\footnotetext{
${ }^{1}$ Project supported by grants from Shanghai Education and Development Foundation (03SG06) and National Natural Science Foundation of China (No 30470690).

${ }^{2}$ Correspondence to Dr Ji-jiang WANG.

Phn 86-21-5423-7913.

Fax 86-21-6417-4579.

E-mail wangjj@shmu.edu.cn

Received 2005-07-14

Accepted 2005-08-31
} \\ doi: $10.1111 / \mathrm{j} .1745-7254.2005 .00231 . \mathrm{x}$
}

\begin{abstract}
Aim: To test the effect of orexin-A (hypocretin-1), a neuropeptide synthesized in the lateral hypothalamus and the perifornical area, on the glycinergic inputs and the GABAergic inputs of cardiac vagal neurons (CVN). Methods: The effects of orexin-A at three concentrations $(20 \mathrm{nmol} / \mathrm{L}, 100 \mathrm{nmol} / \mathrm{L}, 500 \mathrm{nmol} / \mathrm{L})$ on the glycinergic inputs and the GABAergic inputs were investigated by using retrograde fluorescent labeling of cardiac neurons (CVN) in the nucleus ambiguus (NA) and the voltage patch-clamp technique. Results: Orexin-A dose-dependently increased the frequency of both the glycinergic and the GABAergic spontaneous inhibitory postsynaptic currents (sIPSC). However, at a lower concentration $(20 \mathrm{nmol} / \mathrm{L})$ of orexin-A, although the frequency of the glycinergic sIPSC was significantly increased, the frequency of the GABAergic sIPSC was not significantly changed. Conclusion: The glycinergic inputs and the GABAergic inputs have different sensitivities to orexin-A, which suggests that the two kinds of inhibitory inputs might play different roles in the synaptic control of cardiac vagal functions.
\end{abstract}

\section{Introduction}

Orexin-A and orexin-B, also known as hypocretin-1 and hypocretin-2, are members of a new family of neuropeptides synthesized in the lateral hypothalamus and perifornical area neurons ${ }^{[1,2]}$. Orexins have been shown to participate in many functions, including sleep-wakefulness, feeding, and neuroendocrine function ${ }^{[3-5]}$. In addition, many studies have found that orexins also participate in the neural control of cardiovascular functions. And, although most studies have focused on the excitatory effect of orexins on cardiovascular sympathetic activity ${ }^{[6-11]}$, some evidence suggests that orexins might also have a role in the parasympathetic control of heart rate and cardiac functions.

Cardiac vagal nerves predominate over cardiac sympathetic nerves in the control of heart rate and cardiac functions, and their preganglionic fibers primarily originate from the cardiac vagal neurons $(\mathrm{CVN})$ in the nucleus ambiguus (NA) and the dorsal motor nucleus of the vagus (DMNX) ${ }^{[12-15]}$. CVN are intrinsically silent and their activity relies completely on their synaptic inputs ${ }^{[15]}$. CVN receive excitatory gluta- matergic and cholinergic inputs ${ }^{[16,17]}$, and inhibitory GABAergic and glycinergic inputs ${ }^{[1,17,18]}$. Although previous studies have suggested that orexins are involved in the vagal control of heart rate and cardiac functions, the reported effects are largely contradictory. Microinjection of orexin-A into the NA of adult male rats elicited a dose-related decrease in heart rate ${ }^{[19]}$. Microinjection of orexin-A into the nucleus tractus solitarius (NTS), where GABAergic neurons and glutamatergic neurons monosynaptically project to $\mathrm{CVN}^{[14,16]}$, elicited a decrease ${ }^{[20]}$ or increase ${ }^{[21]}$ in heart rate. In a very recent in vitro study using brainstem slices of newborn rats, orexin-A was found to inhibit CVN indirectly, via presynaptic enhancement of the actionpotential-dependent GABAergic and glycinergic inputs, and via presynaptic attenuation of the actionpotential-dependent glutamatergic inputs $^{[22]}$.

The actual concentration of orexin-A in rat brainstem that acts to modulate the physiological synaptic control of $\mathrm{CVN}$ is not clear; however, the experimentally measured concentration of orexin-A in the cerebrospinal fluid is less than $1 \mathrm{nmol} / \mathrm{L}$ in $\operatorname{rats}^{[23]}$, and is even less in dogs and human 
beings ${ }^{[24,25]}$. Surprisingly, the concentration of orexin-A used in the in vitro study of Dergacheva et al ${ }^{[22]}$ was $1 \mu \mathrm{mol} / \mathrm{L}$, and was as high as $1 \mu \mathrm{mol} / \mathrm{L}$ in microinjection studies ${ }^{[19-21]}$. It is possible that these previous studies have used overdoses of orexin-A, which might have resulted in a non-specific effect.

The response of each kind of synaptic input of CVN to orexin-A might be different at different concentrations. At the same concentration, the sensitivity of each kind of synaptic input to orexin-A might also be different. These possibilities make it necessary to reevaluate the effect of orexin-A on $\mathrm{CVN}$ at lower and more divided concentrations. The purpose of the present study is to reevaluate the effect of orexinA on the glycinergic and GABAergic spontaneous inhibitory postsynaptic currents (sIPSC) at lower concentrations (20 nmol/L, $100 \mathrm{nmol} / \mathrm{L}$, and $500 \mathrm{nmol} / \mathrm{L})$. Using retrograde fluorescent labeling of CVN and the voltage patch-clamp technique, we have demonstrated that orexin-A dose-dependently increases the frequency of both the glycinergic and the GABAergic sIPSC. However, at a lower concentration $(20 \mathrm{nmol} / \mathrm{L})$ of orexin-A, although the frequency of the glycinergic sIPSC was significantly increased, the frequency of the GABAergic sIPSC was not significantly changed. These results suggest that the glycinergic inputs and the GABAergic inputs have different sensitivities to orexin-A, and suggest that the two kinds of inhibitory inputs might play different roles in the synaptic control of cardiac vagal functions.

\section{Materials and methods}

Retrograde fluorescent labeling of $\mathrm{CVN}$ The inhalation agent halothane $(0.5 \mathrm{~mL})$ was dripped into a glass box $(5 \mathrm{~cm} \times 5 \mathrm{~cm} \times 5 \mathrm{~cm})$ with a lid and a bottom cotton pad. Three to four-day-old Sprague-Dawley rats (Shanghai Institute for Family Planning) were put in the box for $30 \mathrm{~s}$ with the lid covered. This procedure anesthetized the rats but maintained their breathing in a relatively normal state. The rats were then buried in ice-filled bags to decrease the rats' body temperatures and slow their hearts. After autonomic breathing stopped (usually within $2 \mathrm{~min}$ ) a right thoracotomy was carried out to expose the heart, and rhodamine ( $2 \%$ solution, 20-50 $\mu \mathrm{L}$; XRITC, Molecular Probes, Carlsbad, California, USA) was injected into the pericardial sac with a glass pipette (tip diameter $50 \mu \mathrm{m}$ ). The incision was sutured and the animals were heated with a thermo-pad to help recovery. After the surgery (about $5 \mathrm{~min}$ ) the animals usually started autonomic breathing within $3 \mathrm{~min}$ and started free moving within another $5 \mathrm{~min}$. The animals were allowed $1-2 \mathrm{~d}$ to recover, and experiments were performed when animals were 4-6 d old. Rats at this age have been shown to have similar respiratory-related and reflex-related parasympathetic heart rate control as adult rats ${ }^{[17,26]}$. Similar surgical procedures that selectively labeled CVN have been described previously by Mendelowitz and Kunze ${ }^{[27]}$, who proved that no brainstem neurons were labeled if rhodamine was injected into the chest cavity while the pericardial sac was kept intact, and if rhodamine was injected into the pericardial sac while the cardiac branch of the cardiac vagal nerve was sectioned. In addition, it has also been proved that intravenous injection of up to 10 $\mathrm{mg}$ of rhodamine failed to label any $\mathrm{CVN}^{[27]}$.

Slice preparation The animals were anesthetized deeply with halothane and decapitated at the supracollicular level. The hindbrain was exposed, isolated, and submerged in cold $\left(4{ }^{\circ} \mathrm{C}\right)$ artificial cerebral spinal fluid (ACSF) of the following composition (in mmol/L): $\mathrm{NaCl}$ (124), $\mathrm{KCl}(3.0), \mathrm{KH}_{2} \mathrm{PO}_{4}(1.2)$, $\mathrm{CaCl}_{2}$ (2.4), $\mathrm{MgSO}_{4}$ (1.3), $\mathrm{NaHCO}_{3}$ (26), D-glucose (10), sucrose (10), and constantly bubbled with gas $\left(95 \% \mathrm{O}_{2}, 5 \%\right.$ $\mathrm{CO}_{2} ; \mathrm{pH}$ 7.4). The cerebellum was removed and the brainstem was dissected using a dissection microscope. With the rostral end facing upwards and the ventral surface facing the razor, the brainstem was then secured in the slicing chamber of a vibratome (Leica VT $1000 \mathrm{~S}$, Heerbrugg, Switzerland) filled with the same ACSF, and sequentially sectioned in variable thickness in the transverse plane. Once the NA and other landmarks ${ }^{[17]}$ emerged under the microscope, a single slice of $400 \mu \mathrm{m}$ thickness was taken for experimentation. A medulla slice of this thickness from a 4-6-d-old rat actually includes the full range of the medulla, and such slices have been used in many studies (eg, respiratory rhythm studies) that require relatively intact in vitro neuronal networks. The slice was transferred into the recording chamber and submerged in the ACSF maintained at $22^{\circ} \mathrm{C}$.

Electrophysiological recording Individual CVN in the NA were identified by the presence of the fluorescent tracer using an Olympus (Tokyo, Japan) upright microscope through a $40 \times$ water immersion objective. These identified CVN were then imaged with differential interference contrast (DIC) optics, and infrared illumination and infrared-sensitive video detection cameras to gain better spatial resolution and to visually guide and position the patch pipette onto the surface of the identified neuron. The pipette $(2.0-5.0 \Omega)$ was advanced until a high resistance seal was obtained $(>1 \mathrm{G} \Omega$ ) between the pipette tip and the cell membrane of the identified neuron. The membrane under the pipette tip was then ruptured with a brief suction to obtain whole-cell patch-clamp configuration, and the cell was voltage-clamped at a holding potential of $-80 \mathrm{mV}$. The pipette resistance and capacitance 
was not compensated either before or after gaining intracellular access. To record GABAergic and glycinergic synaptic events, the patch pipettes were filled with a solution consisting of (in mmol/L): $\mathrm{KCl}(150), \mathrm{MgCl}_{2}$ (2), ethyleneglycolbis( $\beta$-aminoethyl ether)- $N, N, N^{\prime}, N^{\prime}$-tetraacetic acid (EGTA)(2), $N$-2-hydroxyethylpiperazine- $N$ '-2-ethanesulfonic acid (HEPES) (10), Mg-ATP (2), at $\mathrm{pH} 7.35$. With this pipette solution the $\mathrm{Cl}^{-}$current induced by activation of the GABA ( $\gamma$-aminobutyric acid) receptors and/or glycinergic receptors was recorded as an inward current. In some experiments a $2 \mathrm{~ms}, 5 \mathrm{mV}$, hyperpolarizing current was injected with a frequency of $0.25 \mathrm{~Hz}$ to calculate and monitor the membrane resistance change of CVN throughout the recording. All animal procedures complied with the institutional guidelines of Fudan University, and were in accordance with the National Administration Guidelines for Experimental Animals.

Drug application GABAergic synaptic currents were isolated by inclusion in a perfusate of strychnine $(1 \mu \mathrm{mol} / \mathrm{L})$, D-2-amino-5-phosphonovalerate $\left(\mathrm{AP}_{5}, 50 \mu \mathrm{mol} / \mathrm{L}\right)$, and 6cyano-7-nitroquinoxaline-2,3-dione (CNQX; $50 \mu \mathrm{mol} / \mathrm{L}$ ) to block glycine, NMDA ( $N$-methyl- $D$-aspartate), and nonNMDA receptors, respectively, and were confirmed by blockade of the synaptic currents by $1 \mu \mathrm{mol} / \mathrm{L}$ picrotoxin at the end of the experiment. Glycinergic synaptic currents were isolated by inclusion in a perfusate of picrotoxin $(1 \mu \mathrm{mol} / \mathrm{L})$, $\mathrm{AP}_{5}(50 \mu \mathrm{mol} / \mathrm{L})$, and CNQX $(50 \mu \mathrm{mol} / \mathrm{L})$ to block GABA, NMDA, and non-NMDA receptors, respectively, and were confirmed by blockade of the synaptic currents by $1 \mu \mathrm{mol} / \mathrm{L}$ strychnine at the end of the experiment. Orexin-A $(20 \mathrm{nmol} / \mathrm{L}$, $100 \mathrm{nmol} / \mathrm{L}, 500 \mathrm{nmol} / \mathrm{L}$ ) was used to activate the orexin-A receptors. The duration of orexin-A application was $2 \mathrm{~min}$ and each slice was applied only once to avoid desensitization. All drugs were purchased from Sigma Aldrich (St Louis, MO, USA).

Data analysis Spontaneous GABAergic and glycinergic sIPSC were analyzed with MiniAnalysis (Synaptosoft, version 4.3.1, Decatur, GA, USA) with a minimal acceptable amplitude of $10 \mathrm{pA}$. Results are presented as mean $\pm \mathrm{SEM}$, and are statistically compared by using the nonparametric Kolmogorov-Smirnov (K-S) test. Significant difference was set at $P<0.05$.

\section{Results}

Responses of the glycinergic sIPSC to different doses of orexin-A Orexin-A significantly increased the frequency of the glycinergic sIPSC at all three concentrations tested. At a concentration of $20 \mathrm{nmol} / \mathrm{L}$ orexin-A increased the average frequency of the glycinergic sIPSC by $63 \% \pm 4 \%$, from
$3.04 \pm 1.01 \mathrm{~Hz}$ to $4.97 \pm 0.91 \mathrm{~Hz}(P<0.01, n=6)$. This frequency increase was observed in all the $6 \mathrm{CVN}$ tested. At a concentration of $100 \mathrm{nmol} / \mathrm{L}$, orexin-A increased the average frequency of the glycinergic sIPSC by $167 \% \pm 5 \%$, from $3.60 \pm$ $0.90 \mathrm{~Hz}$ to $9.64 \pm 0.86 \mathrm{~Hz}(P<0.01, n=8)$. At a concentration of $500 \mathrm{nmol} / \mathrm{L}$ orexin-A caused a frequency increase by $149 \% \pm 7 \%$, from $3.55 \pm 1.21 \mathrm{~Hz}$ to $8.85 \pm 2.71 \mathrm{~Hz}(P<0.05, n=8)$, which was similar in degree to that produced by $100 \mathrm{nmol} / \mathrm{L}$ orexin-A. These results indicate that orexin-A dose-dependently increases the frequency of the glycinergic sIPSC, and a maximal effect can be achieved at $100 \mathrm{nmol} / \mathrm{L}$. A typical CVN with an increased frequency of glycinergic sIPSC induced by orexin-A at $100 \mathrm{nmol} / \mathrm{L}$ is shown in Figure 1A, 1B, and the changes in average frequency caused by the three concentrations of orexin-A are shown in Figure 1C, 1D.

In most CVN and at all the three concentrations of orexinA used, an increase in the amplitude of the glycinergic sIPSC was also observed. Because Dergacheva et al proved that this amplitude increase was presynaptically caused by summated action-potential-dependent release of glycine ${ }^{[22]}$, the amplitude data were not statistically analyzed and were not presented in this article. In addition, at no concentration of orexin-A used in this study was there any significant change in the membrane resistance, or visible change in the baseline currents of CVN. The average membrane resistance before and after $500 \mathrm{nmol} / \mathrm{L}$ orexin-A was $370.24 \pm 16.17 \mathrm{M} \Omega$ and $382.66 \pm 20.87 \mathrm{M} \Omega$, respectively $(P>0.05, n=8)$.

Responses of the GABAergic sIPSC to different doses of orexin-A Orexin-A significantly increased the frequency of the GABAergic sIPSC only at $100 \mathrm{nmol} / \mathrm{L}$ and $500 \mathrm{nmol} / \mathrm{L}$. At a concentration of $20 \mathrm{nmol} / \mathrm{L}$ orexin-A changed the frequency of the GABAergic sIPSC from $3.40 \pm 0.75 \mathrm{~Hz}$ to $2.88 \pm 0.53$ $\mathrm{Hz}(P>0.05, n=6)$. In none of these $6 \mathrm{CVN}$ was obvious frequency change observed. At a concentration of $100 \mathrm{nmol} / \mathrm{L}$ orexin-A increased the frequency of the GABAergic sIPSC by $97 \% \pm 11 \%$, from $2.58 \pm 0.63 \mathrm{~Hz}$ to $5.07 \pm 1.22 \mathrm{~Hz}(P<0.05, n=6)$. At a concentration of $500 \mathrm{nmol} / \mathrm{L}$ orexin-A increased the frequency of the GABAergic sIPSC by $173 \% \pm 14 \%$, from $2.59 \pm 0$. $82 \mathrm{~Hz}$ to $7.08 \pm 0.86 \mathrm{~Hz}(P<0.01, n=5)$. These results indicate that although orexin-A also dose-dependently increased the frequency of the GABAergic sIPSC, a relatively higher concentration $(>20 \mathrm{nmol} / \mathrm{L})$ is needed to cause a significant change, and the maximal frequency increase could not be reached at a concentration of $100 \mathrm{nmol} / \mathrm{L}$. A typical CVN with an increased frequency of the GABAergic sIPSC induced by orexin-A at $100 \mathrm{nmol} / \mathrm{L}$ is shown in Figure 2A, 2B, and the changes in average frequency caused by the three concentrations of orexin-A are illustrated in Figure 2C, 2D.

Orexin-A at concentrations of $100 \mathrm{nmol} / \mathrm{L}$ and $500 \mathrm{nmol} / \mathrm{L}$ 
A
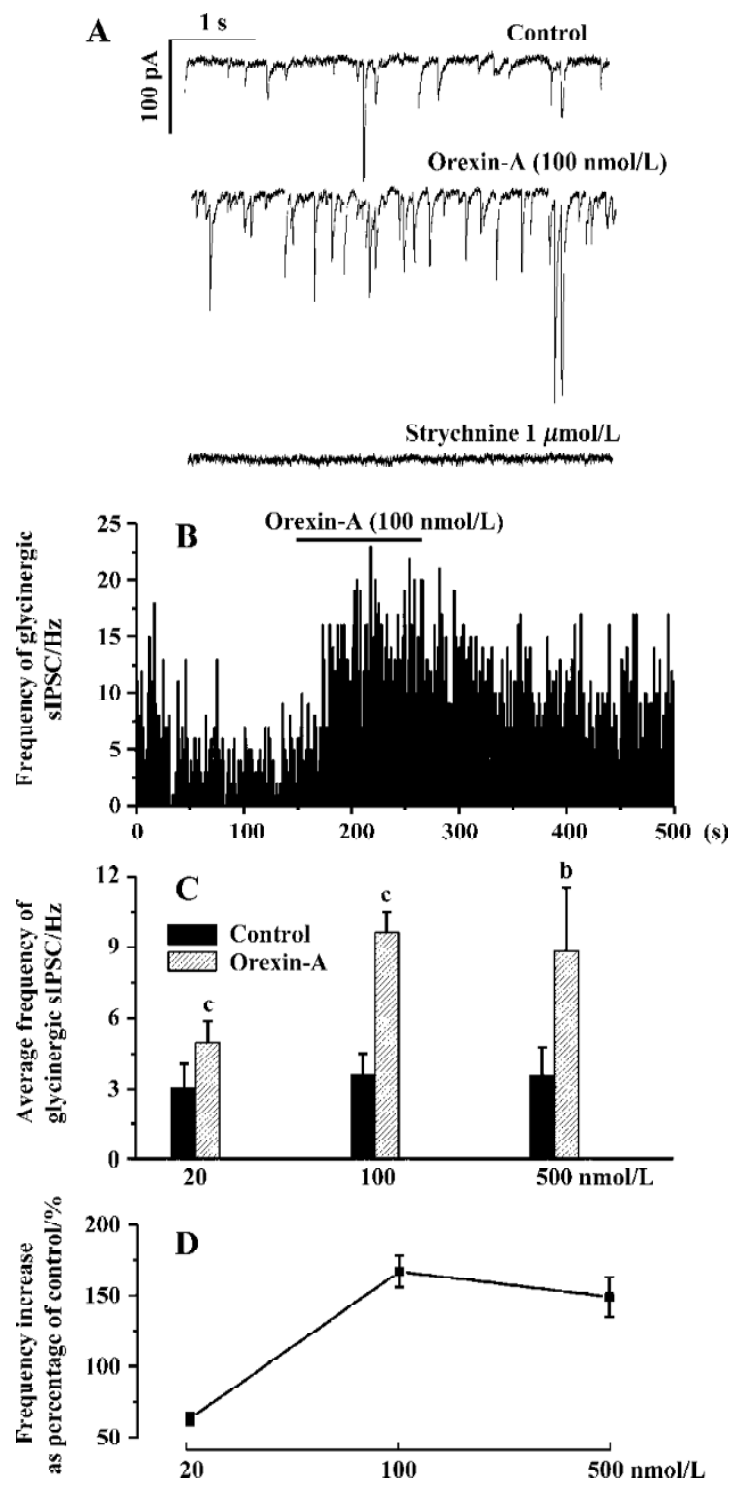

Figure 1. Orexin-A dose-dependently increased the frequency of the glycinergic sIPSC. (A) Traces that show the glycinergic sIPSC during control, during application of $100 \mathrm{nmol} / \mathrm{L}$ orexin-A and during application of $1 \mu \mathrm{mol} / \mathrm{L}$ strychnine, respectively. (B) Frequency histogram that shows the frequency increase of the glycinergic sIPSC during application of $100 \mathrm{nmol} / \mathrm{L}$ orexin-A in a representative CVN. (C) The increase in the average frequency of the glycinergic sIPSC during application of $20 \mathrm{nmol} / \mathrm{L}, 100 \mathrm{nmol} / \mathrm{L}$, and $500 \mathrm{nmol} / \mathrm{L}$ orexinA. ${ }^{\mathrm{b}} P<0.05,{ }^{\mathrm{c}} P<0.01 \mathrm{vs}$ control. (D) Frequency increase of the glycinergic sIPSC as a percentage of control caused by orexin-A at 20 $\mathrm{nmol} / \mathrm{L}, 100 \mathrm{nmol} / \mathrm{L}$, and $500 \mathrm{nmol} / \mathrm{L}$.

also caused an amplitude increase of the GABAergic sIPSC in most CVN tested. Because Dergacheva et al have proved that this amplitude increase is also presynaptically caused by summated action-potential-dependent release of GABA $^{[22]}$ the amplitude data were not statistically ana-
A
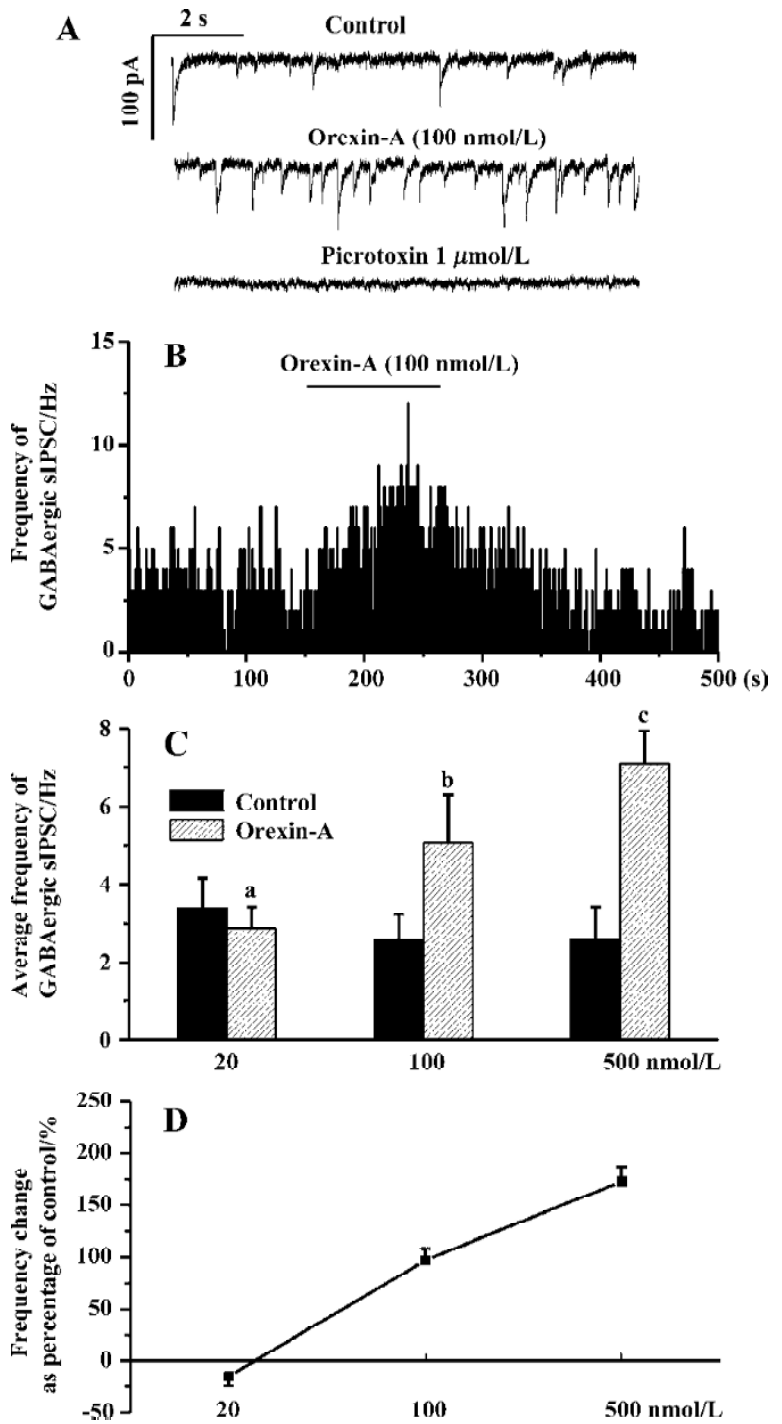

Figure 2. Orexin-A dose-dependently increased the frequency of the GABAergic sIPSC. (A) Traces that show the GABAergic sIPSC during control, during application of $100 \mathrm{nmol} / \mathrm{L}$ orexin-A and during application of $1 \mu \mathrm{mol} / \mathrm{L}$ picrotoxin, respectively. (B) Frequency histogram that shows the frequency increase of the GABAergic sIPSC during application of $100 \mathrm{nmol} / \mathrm{L}$ orexin-A in a representative CVN. (C) The increase in the average frequency of the GABAergic sIPSC during application of $20 \mathrm{nmol} / \mathrm{L}, 100 \mathrm{nmol} / \mathrm{L}$, and $500 \mathrm{nmol} / \mathrm{L}$ orexinA. ${ }^{\mathrm{a} P} P 0.05,{ }^{\mathrm{b}} P<0.05,{ }^{\mathrm{c}} P<0.01 \mathrm{vs}$ control. (D) Frequency change of the GABAergic sIPSC as a percentage of control caused by orexin-A at $20 \mathrm{nmol} / \mathrm{L}, 100 \mathrm{nmol} / \mathrm{L}$, and $500 \mathrm{nmol} / \mathrm{L}$.

lyzed and are not presented in this article.

\section{Discussion}

There are two major findings in the present study. One is that the frequency increase of both the glycinergic and the GABAergic IPSC caused by orexin-A is dose-dependent. The 
other is that orexin-A at a lower concentration $(20 \mathrm{nmol} / \mathrm{L})$ significantly enhances the glycinergic inputs but does not cause significant alteration in the GABAergic inputs.

Ever since the discovery of the GABAergic inputs and the glycinergic inputs in CVN, these two inhibitory inputs have shown identical roles in the regulation of CVN. The synaptic activities of both kinds of inputs are action-potential-dependent and are absent in the presence of tetrodotoxin (TTX $)^{[14,18]}$; both kinds of inputs are enhanced by endogenous acetylcholine and exogenous nicotine ${ }^{[18]}$, are inhibited by opioids, and are rhythmically augmented during inspiration ${ }^{[17,28]}$. Little is known about whether and how these two inhibitory inputs play different roles in the synaptic control of CVN, except that dihydro- $\beta$-erythroidine, a nicotinic antagonist, prevents the inspiratory-related augmentation of the GABAergic, but not the glycinergic, inputs to CVN when focally applied at $3 \mathrm{mmol} / \mathrm{L}$, a concentration under which dihydro- $\beta$-erythroidine specifically binds to the $\alpha 4 \beta 2$ type of nicotinic receptors ${ }^{[17]}$. The present study demonstrated that orexin-A at a lower concentration $(20 \mathrm{nmol} / \mathrm{L})$ significantly increased the frequency of the glycinergic sIPSC, but did not cause a significant frequency alteration in the GABAergic sIPSC. These results indicate that the glycinergic inputs and GABAergic inputs might have different roles in the orexinergic control of CVN. At lower concentrations ( $\leq 20 \mathrm{nmol} / \mathrm{L})$ of orexin-A, glycinergic inputs might dominate, and at higher concentrations ( $\geq 20 \mathrm{nmol} / \mathrm{L})$ of orexin-A, a synergistic action of both kinds of inputs might occur.

Although in the present study lower concentrations of orexin-A were used, the results were still quite consistent with those from the study of Dergacheva et $a l^{[22]}$. The results of the present study as well as the results of Dergacheva et al indicate that the responses of the glycinergic inputs and the GABAergic inputs to different concentrations of orexin-A differ in degree, but do not differ in the direction of responses. The present study still cannot reveal why CVN are excited by microinjection of orexin-A into the NA in vivo and are inhibited in vitro. Further study is necessary.

The physiological roles of the glycinergic and the GABAergic inputs to CVN in cardiac vagal control are not fully understood. Currently, the only confirmed function of these two inputs to $\mathrm{CVN}$ is that they are involved in the generation of respiratory-related heart rate rhythm. During the inspiratory phase of the respiratory cycle, both the GABAergic and the glycinergic inputs are facilitated by acetylcholine to inhibit CVN and speed up the heart ${ }^{[18,27]}$. These two inhibitory inputs to CVN have also long been postulated to be involved in the reflex parasympathetic control of the heart. The serotonergic neurons in the brainstem or in the nodose ganglion have been reported to be activated during activation of the cardiovascular chemo- or baro-reflex ${ }^{[29]}$. The activation of the serotonergic system, via inhibition of both the GABAergic and the glycinergic inputs to CVN, possibly excites CVN and mediates the reflex parasympathetic inhibition of the heart. The physiological significance of the modulation of the GABAergic and the glycinergic inputs to CVN by orexin-A is also not clear, and nothing is known about why the glycinergic neurons preceding $\mathrm{CVN}$ were more sensitive to orexin-A than the GABAergic neurons. Perhaps the receptors for orexin-A binding in these two kinds of neurons are different in density. Orexin-A is known to strengthen preying behavior and increases food uptake. Because the action of orexin-A on CVN is assumed to accelerate the heart, orexin-A might help mammals to adapt to their preying and eating behavior via speeding up the heart.

In conclusion, the present study demonstrated that orexin-A dose-dependently increased the frequency of both the glycinergic and the GABAergic sIPSC of CVN; the glycinergic neurons and the GABAergic neurons preceding CVN had different sensitivities to orexin-A, which might indicate that these two kinds of inhibitory neurons play different roles in the synaptic control of cardiac vagal functions.

\section{References}

1 de Lecea L, Kilduff T, Peyron C, Gao X, Foye PE, Danielson PE, et al. The hypocretins: hypothalamus-specific peptides with neuroexcitatory activity. Proc Natl Acad Sci USA 1998; 95: 322-7.

2 Sakurai T, Amemiya A, Ishii M, Matsuzaki I, Chemelli RM, Tanaka $\mathrm{H}$, et al. Orexins and orexin receptors: a family of hypothalamic neuropeptides and $\mathrm{G}$ protein-coupled receptors that regulate feeding behavior. Cell 1998; 92: 573-85.

3 Burdakov D, Liss B, Ashcroft FM. Orexin excites GABAergic neurons of the arcuate nucleus by activating the sodium-calcium exchanger. J Neurosci 2003; 23: 4951-7.

4 van den Pol AN, Gao XB, Obrietan K, Kilduff TS, Belousov AB. Presynaptic and postsynaptic actions and modulation of neuroendocrine neurons by a new hypothalamic peptide, hypocretin/ orexin. J Neurosci 1998; 18: 7962-71.

5 Wu M, Zhang Z, Leranth C, Xu C, van den Pol AN, Alreja M. Hypocretin increases impulse flow in the septohippocampal GABAergic pathway: implications for arousal via a mechanism of hippocampal disinhibition. J Neurosci 2002; 22: 7754-65.

6 Matsumura K, Tsuchihashi T, Abe I. Central orexin-A augments sympathoadrenal outflow in conscious rabbits. Hypertension 2001; 37: 1382-7.

7 Samson WK, Gosnell B, Chang JK, Resch ZT, Murphy TC. Cardiovascular regulatory actions of the hypocretins in brain. Brain Res 1999; 831: 248-53.

8 Shirasaka T, Nakazato M, Matsukura S, Takasaki M, Kannan H. 
Sympathetic and cardiovascular actions of orexins in conscious rats. Am J Physiol 1999; 277: R1780-5.

9 Antunes VR, Brailoiu GC, Kwok EH, Scruggs P, Dun NJ. Orexins/ hypocretins excite rat sympathetic preganglionic neurons in vivo and in vitro. Am J Physiol Regul Integr Comp Physiol 2001; 281: R1801-7.

10 Chen CT, Hwang LL, Chang JK, Dun NJ. Pressor effects of orexins injected intracisternally and to rostral ventrolateral medulla of anesthetized rats. Am J Physiol Regul Integr Comp Physiol 2000; 278: R692-7.

11 Machado BH, Bonagamba LG, Dun SL, Kwok EH, Dun NJ. Pressor response to microinjection of orexin/hypocretin into rostral ventrolateral medulla of awake rats. Regul Pept 2002; 104: 75-81.

12 Cheng Z, Powley TL. Nucleus ambiguus projections to cardiac ganglia of rat atria: an anterograde tracing study. J Comp Neurol 2000; 424: 588-606.

13 Mendelowitz D. Advances in parasympathetic control of heart rate and cardiac function. News Physiol Sci 1999; 14: 155-61.

14 Wang J, Irnaten M, Mendelowitz D. Characteristics of spontaneous and evoked GABAergic synaptic currents in cardiac vagal neurons in rats. Brain Res 2001; 889: 78-83.

15 Wang J, Irnaten M, Neff RA, Venkatesan P, Evans C, Loewy AD, et al. Synaptic and neurotransmitter activation of cardiac vagal neurons in the nucleus ambiguus. Ann N Y Acad Sci 2001; 940: $237-46$.

16 Neff RA, Mihalevich M, Mendelowitz D. Stimulation of NTS activates NMDA and non-NMDA receptors in rat cardiac vagal neurons in the nucleus ambiguus. Brain Res 1998; 792: 277-82.

17 Neff RA, Wang J, Baxi S, Evans C, Mendelowitz D. Respiratory sinus arrhythmia: endogenous activation of nicotinic receptors mediates respiratory modulation of brainstem cardioinhibitory parasympathetic neurons. Circ Res 2003; 93: 565-72.

18 Wang J, Wang X, Irnaten M, Venkatesan P, Evans C, Baxi S, et al. Endogenous acetylcholine and nicotine activation enhances GABAergic and glycinergic inputs to cardiac vagal neurons. J Neurophysiol 2003; 89: 2473-81.

19 Ciriello J, de Oliveira CV. Cardiac effects of hypocretin-1 in nucleus ambiguus. Am J Physiol Regul Integr Comp Physiol
2003; 284: R1611-20.

20 de Oliveira CV, Rosas-Arellano MP, Solano-Flores LP, Ciriello J. Cardiovascular effects of hypocretin-1 in nucleus of the solitary tract. Am J Physiol Heart Circ Physiol 2003; 284: H1369-77.

21 Smith PM, Connolly BC, Ferguson AV. Microinjection of orexin into the rat nucleus tractus solitarius causes increases in blood pressure. Brain Res 2002; 950: 261-7.

22 Dergacheva O, Wang X, Huang ZG, Bouairi E, Stephens C, Gorini $\mathrm{C}$, et al. Hypocretin 1 (orexin A) facilitates inhibitory and diminishes excitatory synaptic pathways to cardiac vagal neurons in the nucleus ambiguus. J Pharmacol Exp Ther 2005; 314(3): 1322-7.

23 Fujiki N, Yoshida Y, Ripley B, Honda K, Mignot E, Nishino SCA. Changes in CSF hypocretin-1 (orexin A) levels in rats across 24 hours and in response to food deprivation. Neuroreport 2001; 12: 993-7.

24 John J, Wu MF, Maidment NT,Lam HA, Boehmer LN, Patton $\mathrm{M}$, et al. Developmental changes in CSF hypocretin-1 (orexinA) levels in normal and genetically narcoleptic Doberman pinschers. J Physiol 2004; 560: 587-92.

25 Kiyashchenko LI, Mileykovskiy BY, Maidment N, Lam HA, Wu MF, John J, et al. Release of hypocretin (orexin) during waking and sleep states. J Neurosci 2002; 22: 5282-6.

26 Irnaten M, Neff RA, Wang J, Loewy AD, Mettenleiter TC, Mendelowitz D. Activity of cardiorespiratory networks revealed by transsynaptic virus expressing GFP. J Neurophysiol 2001; 85: 435-8.

27 Mendelowitz D, Kunze DL. Identification and dissociation of cardiovascular neurons from the medulla for patch clamp analysis. Neurosci Lett 1991; 132: 217-21.

28 Wang X, Dergacheva O, Griffioen KJ, Huang ZG, Evans C, Gold A, et al. Action of kappa and delta opioid agonists on premotor cardiac vagal neurons in the nucleus ambiguus. Neuroscience 2004; 129: 235-41.

29 Wang, Y, Ramage, AG. The role of central 5-HT (1 A) receptors in the control of B-fibre cardiac and bronchoconstrictor vagal preganglionic neurones in anaesthetized cats. J Physiol (London) 2001; 536: 753-67. 